\title{
Article \\ Reducing Duration of Antibiotic Use for Presumed Neonatal Early-Onset Sepsis in Greek NICUs. A “Low-Hanging Fruit" Approach
}

\author{
Ioannis Kopsidas ${ }^{1,2, *}$, Grammatiki-Christina Tsopela ${ }^{1}$, Nafsika-Maria Molocha ${ }^{1}$, Eleni Bouza ${ }^{3}$, \\ Elisavet Chorafa ${ }^{4}$, Evangelia Chorianopoulou ${ }^{1}$, Vasileios Giapros ${ }^{5}$, Despoina Gkentzi ${ }^{6}{ }^{\mathbb{D}}$, \\ Theodoros Gkouvas ${ }^{7}{ }^{\circledR}$, Anastasia Kapetanaki ${ }^{8}$, Korina Karachristou ${ }^{9}$, Georgia Karavana ${ }^{10}$, Eleni Kourkouni ${ }^{1}$, \\ Georgia Kourlaba ${ }^{1}$, Maria Lithoxopoulou ${ }^{11}$, Vassiliki Papaevangelou ${ }^{12}$, Maria Polychronaki ${ }^{13}$, \\ Emmanuel Roilides ${ }^{4}$, Tania Siahanidou ${ }^{14}{ }^{(0)}$, Evangelia Stratiki ${ }^{15}$, George A. Syrogiannopoulos ${ }^{16}$, \\ Christos Triantafyllou ${ }^{1(D)}$, Maria N. Tsolia ${ }^{2}$, Emmanouela Tsouvala ${ }^{17}{ }^{(}$, Theoklis Zaoutis ${ }^{1,18}$, Nikos Spyridis ${ }^{2}$ (D) \\ and Preventing Hospital-Acquired Infections in Greece (PHiG) Investigators ${ }^{\dagger}$
}

\section{check for}

updates

Citation: Kopsidas, I.; Tsopela, G.-C.; Molocha, N.-M.; Bouza, E.; Chorafa, E.; Chorianopoulou, E.; Giapros, V.; Gkentzi, D.; Gkouvas, T.; Kapetanaki, A.; et al. Reducing Duration of Antibiotic Use for Presumed Neonatal EarlyOnset Sepsis in Greek NICUs. A "Low-Hanging Fruit" Approach. Antibiotics 2021, 10, 275. https:// doi.org/10.3390/antibiotics10030275

Academic Editor: Albert Figueras

Received: 12 February 2021

Accepted: 5 March 2021

Published: 9 March 2021

Publisher's Note: MDPI stays neutral with regard to jurisdictional claims in published maps and institutional affiliations.

Copyright: (c) 2021 by the authors. Licensee MDPI, Basel, Switzerland. This article is an open access article distributed under the terms and conditions of the Creative Commons Attribution (CC BY) license (https:// creativecommons.org/licenses/by/ $4.0 /)$.
1 Center For Clinical Epidemiology and Outcomes Research (CLEO), 11528 Athens, Greece; c.tsopela@cleoresearch.org (G.-C.T.); nmolocha134@gmail.com (N.-M.M.); chorian_lilian@hotmail.com (E.C.); e.kourkouni@cleoresearch.org (E.K.); kurlaba@gmail.com (G.K.); c.triantafyllou@cleoresearch.org (C.T.); zaoutis@email.chop.edu (T.Z.)

2 Infectious Diseases Unit, 2nd Department of Pediatrics, National and Kapodistrian University of Athens (NKUA), 11527 Athens, Greece; mariantsolia@gmail.com (M.N.T.); nspyridis@hotmail.co.uk (N.S.)

3 B' Neonatal Intensive Care Unit, "Aghia Sophia” Children's Hospital, 11527 Athens, Greece; ebouzaat@otenet.gr

4 Third Department of Pediatrics, Aristotle University of Thessaloniki, Hippokration Hospital, 54642 Thessaloniki, Greece; elsachorafa@hotmail.gr (E.C.); roilides@auth.gr (E.R.)

5 Neonatal Intensive Care Unit, University Hospital of Ioannina, 45500 Ioannina, Greece; vgiapros@uoi.gr

6 Patras Medical School, University General Hospital of Patras, 26504 Patra, Greece; gkentzid@gmail.com

7 Neonatal Intensive Care Unit, "Panagiotis \& Aglaia Kyriakou” Children's Hospital, 11528 Athens, Greece; theogkou@yahoo.gr

8 Neonatal Intensive Care Unit, Elenas Venizelou Maternity Hospital, 11521 Athens, Greece; pednancy@hotmail.com

9 A' Neonatal Intensive Care Unit, “Aghia Sophia” Children's Hospital, 11528 Athens, Greece; korina.karachristou@gmail.com

10 Neonatal Intensive Care Unit, General Hospital of Nikaia and Piraeus "Aghios Panteleimon", 18454 Athens, Greece; gogo_kara@yahoo.gr

11 Second Department of Neonatology, Papageorgiou Hospital, Aristotle University of Thessaloniki, 56429 Thessaloniki, Greece; mlithoxopoulou@yahoo.com

12 Third Department of Pediatrics, National and Kapodistrian University of Athens, General University Hospital “Attikon”, 12462 Athens, Greece; vpapaev@gmail.com

13 Neonatal Intensive Care Unit, Venizelio Hospital, 71409 Heraklion, Greece; polichronaki.mp@gmail.com

14 Neonatal Unit of the First Department of Pediatrics, National and Kapodistrian University of Athens, 11528 Athens, Greece; siahan@med.uoa.gr

15 Neonatal Intensive Care Unit, General District Hospital Athens "Alexandra", 11528 Athens, Greece; zstratiki@gmail.com

16 Department of Pediatrics, Faculty of Medicine, School of Health Sciences, University of Thessaly, 41334 Larissa, Greece; gasyrogiannopoulos@gmail.com

17 Neonatal Intensive Care Unit, Neonatal Department, University General Hospital of Alexandroupolis, 68100 Alexandroupoli, Greece; emtsouvala@gmail.com

18 Division of Infectious Diseases and Center for Pediatric Clinical Effectiveness, Children's Hospital of Philadelphia, Philadelphia, PA 19104, USA

* Correspondence: j.kopsidas@cleoresearch.org

+ These are collaborators. For the PHiG Investigators: Fani Anatolitou, George Baroutis, Stavroula Bounta, Susan Coffin, Gabriel Dimitriou, Aikaterini Drougia, Eleni Fanaraki, Theodoros Gouvias, Aikaterini Kaffe, Paraskevi Karagianni, Marina Koropouli, Katerina Lappa, Stefania Maistreli, George Mavrogeorgos, Antonia Moumouletsa, Angeliki Nika, Irini Nikaina, Stamatia Papadopoulou, Anastasia Papageorgiou, Marianna Skordala-Riti, Athanasios Skoutelis, Maria Theodoraki, Christina Thomou, Pinelopi Triantafyllidou, Asimina Tsintoni, Sotirios Tsiodras, Adamantios Vontzalidis, Kirikas Zannikos. 


\begin{abstract}
Antibiotics are commonly prescribed in Neonatal Intensive Care Units (NICU), where stewardship interventions are challenging. Lowering antibiotic consumption is desperately needed in Greece, a country with high antibiotic resistance rates. We sought to assess the effectiveness of a low-cost and -resource intervention to reduce antibiotic use in Greek NICUs implementing a "lowhanging fruit" approach. A prospective quasi-experimental study was conducted in 15/17 public NICUs in Greece (9/2016-06/2019). The intervention selected was discontinuation of antibiotics within 5 days for neonates with gestational age $\geq 37$ weeks, no documented signs or symptoms of sepsis, CRP $\leq 10 \mathrm{mg} / \mathrm{L}$ and negative cultures within 3 days of antibiotic initiation. Impact was evaluated by the percentage of discontinued regimens by day 5 , length of therapy (LOT) and stay. Trends of antibiotic consumption were assessed with days of therapy (DOT) per 1000 patient-days. Overall, there was a $9 \%$ increase $(p=0.003)$ of antibiotic discontinuation in $\leq 5$ days. In total, $7 / 13(53.8 \%)$ units showed $\mathrm{a} \geq 10 \%$ increase. Overall, 615 days on antibiotics per 1000 patients were saved. Interrupted time-series analysis established a declining trend in DOT/1000 patient-days relative to the pre-intervention trend $(p=0.002)$; a monthly decrease rate of 28.96 DOT $/ 1000$ patient-days ( $p=0.001$, $95 \%$ CI $[-45.33,-12.60])$. The intervention had no impact on antibiotic choice. Antibiotic use was successfully reduced in Greek NICUs using a "low-hanging fruit" approach. In resource-limited settings, similar targeted stewardship interventions can be applied.
\end{abstract}

Keywords: early discontinuation; antibiotic stewardship; prolonged duration; empiric treatment; negative cultures; neonatal intensive care

\title{
1. Introduction
}

Antibiotic resistance is considered one of the most serious threats to global public health and is associated with increased morbidity, mortality and healthcare costs. Leading public health organizations around the world agree that among other actions, antimicrobial stewardship interventions can lead to the reduction of antibiotic use, a key driver in the evolution of resistance to different classes of antimicrobials [1].

Among European nations, Greece ranks highly in terms of antibiotic consumption and rates of antimicrobial resistance [2,3]. The latter is linked to notable disabilities, as well as deaths $[4,5]$. The burden is highest in infants (aged $<1$ year) and people $>65$ years, with an increasing trend compared to 2007 [5].

Antibiotics are the most commonly prescribed medications in Neonatal Intensive Care Units (NICUs) due to several predisposing factors associated with this group of patients, such as natural susceptibility to infections, prematurity, and birth-related complications, as well as postpartum events [6]. Variation in antibiotic use is also common among different NICUs [7], indicating the lack of robust evidence on appropriate indication for initiation, dosing and duration of therapy. Concurrently, there is sufficient evidence to support the link between broad spectrum antibiotic use and adverse outcomes like necrotizing enterocolitis [8,9]. Although blood culture is the gold standard for diagnosing neonatal sepsis, physicians often treat neonates with sterile cultures, despite increasing evidence that unnecessary or prolonged regimens can be harmful [10]. This represents a clear target for improvement in antibiotic use, as it has been estimated that antibiotics for culture-negative sepsis are consumed at 10 times the rate of culture-proven sepsis [11,12].

Initiating antibiotic therapy in neonates is often driven by personal judgement rather than identifying or excluding infection following practice guidelines [12], indicating that stewardship interventions could potentially focus on more straightforward objectives ("low-hanging fruit approach") $[13,14]$ such as stopping antibiotics early. Culture-negative early-onset sepsis is a factor contributing to high antibiotic consumption in NICUs [15].

The primary aim of this study was to assess the effectiveness of a structured intervention in order to reduce duration of antimicrobial use for culture-negative early-onset sepsis across Greek NICUs. The intervention was based on a "low-hanging fruit" approach of antibiotic stewardship practices, so that participating units could enroll patients using 
the existing medical personnel and no additional financial resources. A positive outcome could be an indication that low-cost initiatives can have a significant impact on prudent antibiotic use in different settings such as NICUs.

\section{Results}

A total of 1025 cases of neonates that met the inclusion criteria were identified; 507 in the pre- and 518 in the post-intervention period. Demographic characteristics and risk factors of neonates included in both study periods are listed on Table 1. The majority of them were babies delivered by caesarean section, with unknown maternal GBS status and without prolonged rupture of membranes and no chorioamnionitis.

Table 1. Demographic and clinical characteristics, maternal and neonatal, in the pre- and postintervention period.

\begin{tabular}{|c|c|c|c|}
\hline \multirow{2}{*}{ Number of Neonates \& } & Pre-Intervention & Post-Intervention & $p$-Value \\
\hline & 507 & 518 & \\
\hline Sex & $\mathbf{N}(\%)$ & $\mathbf{N}(\%)$ & \multirow{3}{*}{0.473} \\
\hline Male & $311(61.3)$ & $329(63.5)$ & \\
\hline Female & $196(38.7)$ & $189(36.5)$ & \\
\hline \multicolumn{4}{|l|}{ Delivery } \\
\hline Vaginal & 145 (28.7) & 144 (27.9) & \multirow[t]{2}{*}{0.790} \\
\hline Caesarean & $361(71.3)$ & $372(72.1)$ & \\
\hline \multicolumn{4}{|l|}{ Group B Streptococcus status } \\
\hline Negative & $151(29.8)$ & $132(25.6)$ & \multirow[t]{3}{*}{0.803 * } \\
\hline Positive & $20(4.0)$ & $16(3.1)$ & \\
\hline Unknown & $335(66.2)$ & 367 (71.3) & \\
\hline \multicolumn{4}{|l|}{ Chorioamnionitis } \\
\hline No & $476(94.1)$ & $413(80.3)$ & \multirow[t]{3}{*}{$0.849 *$} \\
\hline Yes & $4(0.8)$ & $3(0.6)$ & \\
\hline Unknown & $26(5.1)$ & $98(19.1)$ & \\
\hline \multicolumn{4}{|l|}{ Rupture of Membranes (>18 h) } \\
\hline No & $461(91.1)$ & $393(97.3)$ & \multirow[t]{4}{*}{0.194 * } \\
\hline Yes & $21(4.2)$ & $11(2.7)$ & \\
\hline Unknown & $24(4.7)$ & $0(0.0)$ & \\
\hline & Median (IQR) & Median (IQR) & \\
\hline Gestational Age (weeks) & $38(37-39)$ & $38(37-39)$ & 0.413 \\
\hline Birth Weight (grams) & $3100(2755-3420)$ & $3140(2800-3420)$ & 0.275 \\
\hline
\end{tabular}

\&: neonates started on empiric antibiotics during the first 3 days of life, with a gestational age $\geq 37$ weeks, no documented signs or symptoms or CRP $\geq 10 \mathrm{mg} / \mathrm{L}$ during the first 3 days of life, and negative cultures taken within 3 days. ${ }^{*} p$-value represents the differences between negative and positive or yes and no.

\subsection{Impact on Length of Therapy and on Discontinuation in 5 Days or Less}

Pre-intervention data showed considerable variation in LOT for these neonates, with the median duration of antibiotic administration ranging from 2 days (IQR 2-3) to 7.5 days (IQR 6-10) across the units (Table 2). Thirteen out of the 15 units continued into the intervention phase. There was a $9 \%$ increase $(p=0.003)$ in the number of neonates that received antibiotics for five or less days in the post intervention period; from $52.5 \%$ $(266 / 507)$ in the pre- to $61.5 \%(319 / 518)$ in the post-intervention period. Examining the changes in each unit separately (Figure 1), there was $a \geq 10 \%$ increase in discontinued regimens by day 5 in $7 / 13(53.8 \%)$ of the units. Nonetheless, in three units, a $\geq 10 \%$ decrease was observed in discontinued regimens in the post-intervention period (Figure 1). Overall, 615 days of antibiotics per 1000 patients were saved during the 15 months of the post-intervention period.

The interrupted time series analysis established no significant trend prior to intervention $(p=0.535)$. However, in the post-intervention period, a decline trend was observed in the DOT/1000 patient-days relative to the pre-intervention trend $(p=0.002)$, lead- 
ing to a monthly decrease rate of 28.96 days of therapy/1000 patient-days ( $p=0.001$, $95 \% \mathrm{CI}=[-45.33,-12.60]$ ) (Figure 2).

Table 2. Length of therapy before and after the intervention of neonates that met the inclusion criteria and given empiric treatment for possible early-onset sepsis.

\begin{tabular}{|c|c|c|c|c|c|c|c|c|}
\hline Unit & $\mathbf{N}_{1}$ & $\operatorname{Mean}_{1}$ (SD) & Median (IQR) & $\mathbf{N}_{2}$ & Mean $_{2}$ (SD) & Median (IQR) & $\begin{array}{c}\text { Difference of Mean AB } \\
\text { Duration before and after } \\
\text { the Intervention }\end{array}$ & $\begin{array}{c}\text { Calculated total } \\
\text { Difference of Antibiotic } \\
\text { Administration Days * }\end{array}$ \\
\hline NICU 1 & 21 & $5.7(4)$ & $5(3-6)$ & 12 & $4.6(1.7)$ & $5(3-5)$ & -1.1 & -13.2 \\
\hline NICU 2 & 51 & $5.5(3.4)$ & $4(3-7)$ & 88 & $4.2(1.5)$ & $4(3-5)$ & -1.3 & -114.4 \\
\hline NICU 3 & 20 & $5.9(2.3)$ & $5(4-7)$ & 22 & $5.5(2.4)$ & $5(4-6)$ & -0.4 & -8.4 \\
\hline NICU 4 & 30 & $7.8(6.9)$ & $7(5-9)$ & 17 & $6.4(1.8)$ & $8(5-9)$ & -1.4 & -23.8 \\
\hline NICU 5 & 18 & $10.5(8.4)$ & $7(5-10)$ & 21 & $7.6(3.6)$ & $6(5-11)$ & -2.9 & -60.9 \\
\hline NICU 6 & 10 & $2.6(1)$ & $2(2-3)$ & 12 & $3.6(1.3)$ & $3(3-4.5)$ & 1 & 12 \\
\hline NICU 7 & 38 & $5(2.4)$ & $4(3-6)$ & 65 & $5.1(2.6)$ & $5(3-7)$ & 0.1 & 6.5 \\
\hline NICU 8 & 32 & $4.4(1.8)$ & $4(3-5.5)$ & 30 & $5.1(3.6)$ & $4(3-7)$ & 0.7 & 21 \\
\hline NICU 9 & 70 & $4.4(3.1)$ & $4(3-5)$ & 37 & $3.4(1.7)$ & $3(2-4)$ & -1 & -37 \\
\hline NICU 10^ & 55 & $5(1.8)$ & $5(3-6)$ & - & - & - & - & - \\
\hline NICU 11 & 84 & $7.7(5.6)$ & $7(4-10)$ & 73 & $8.7(6.8)$ & $7(5-10)$ & 1 & 73 \\
\hline NICU 12 & 16 & $7.8(2.2)$ & $7.5(7-9.5)$ & 11 & $8(3.3)$ & $6(5-9)$ & 0.2 & 2.2 \\
\hline NICU 13 & 67 & $6.4(2.7)$ & $6(5-7)$ & 77 & $5.1(2.3)$ & $4(3-6)$ & -1.3 & -100.1 \\
\hline NICU 14^ & 43 & $4.7(2.6)$ & $4(3-5)$ & - & - & - & - & - \\
\hline NICU 15 & 50 & $8.7(4.2)$ & $7.5(6-10)$ & 54 & $7.3(3.9)$ & $6(5-7)$ & -1.4 & -75.6 \\
\hline Total & 605 & $6.2(4.2)$ & $5(4-7)$ & 518 & $5.8(3.9)$ & $5(3-7)$ & & -318.7 \\
\hline
\end{tabular}

Mean $_{1}$ : Mean length of therapy before intervention. Mean 2 : Mean length of therapy after Intervention. ${ }^{*}\left(\operatorname{Mean}_{1}-\mathrm{Mean}_{2}\right) \times$ number of neonates meeting intervention criteria in the post-intervention period $\left(\mathrm{N}_{2}\right)$. IQR: Inter-quartile range. ^ unit did not proceed to the intervention phase. AB: antibiotic.

Percent of discontinued antibiotic regimens within 5 days

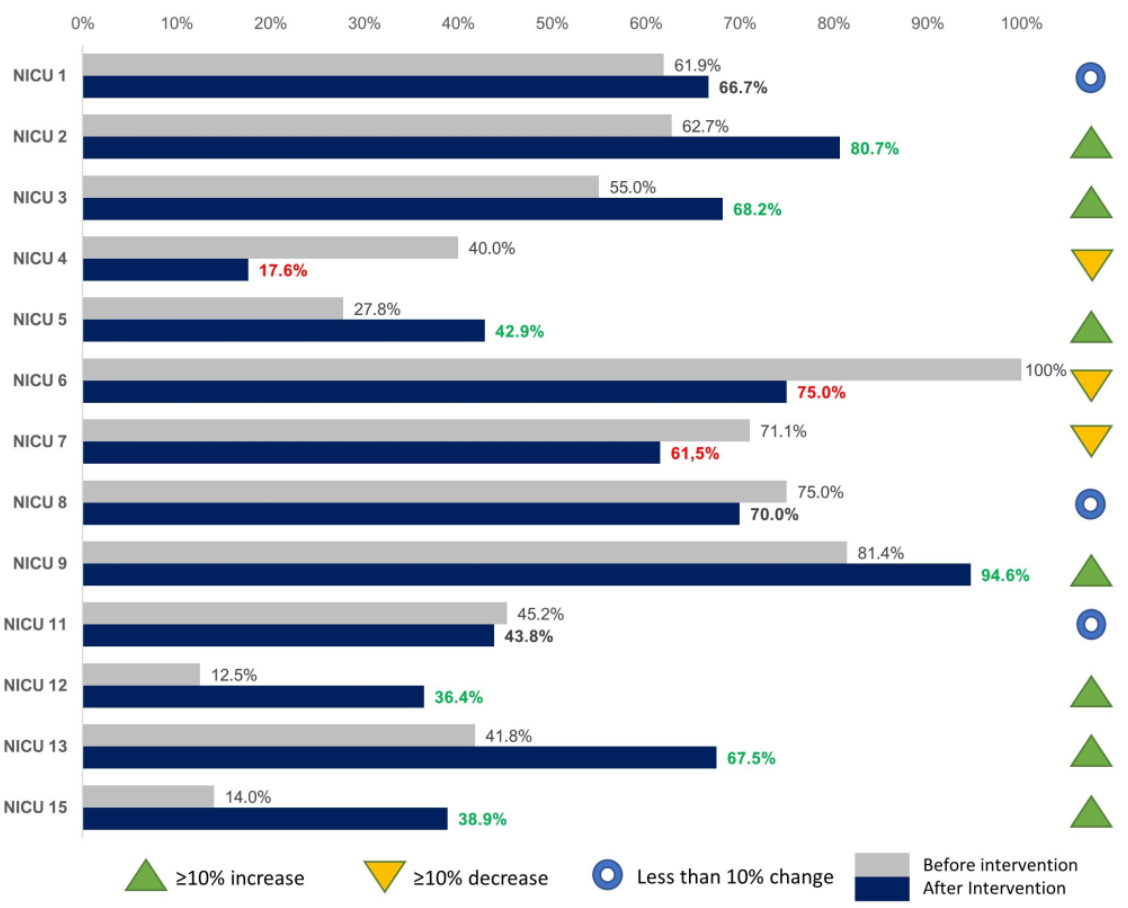

Figure 1. Percent of neonates in each unit that met inclusion criteria and discontinued antibiotics within 5 days of initiation of empiric treatment. 


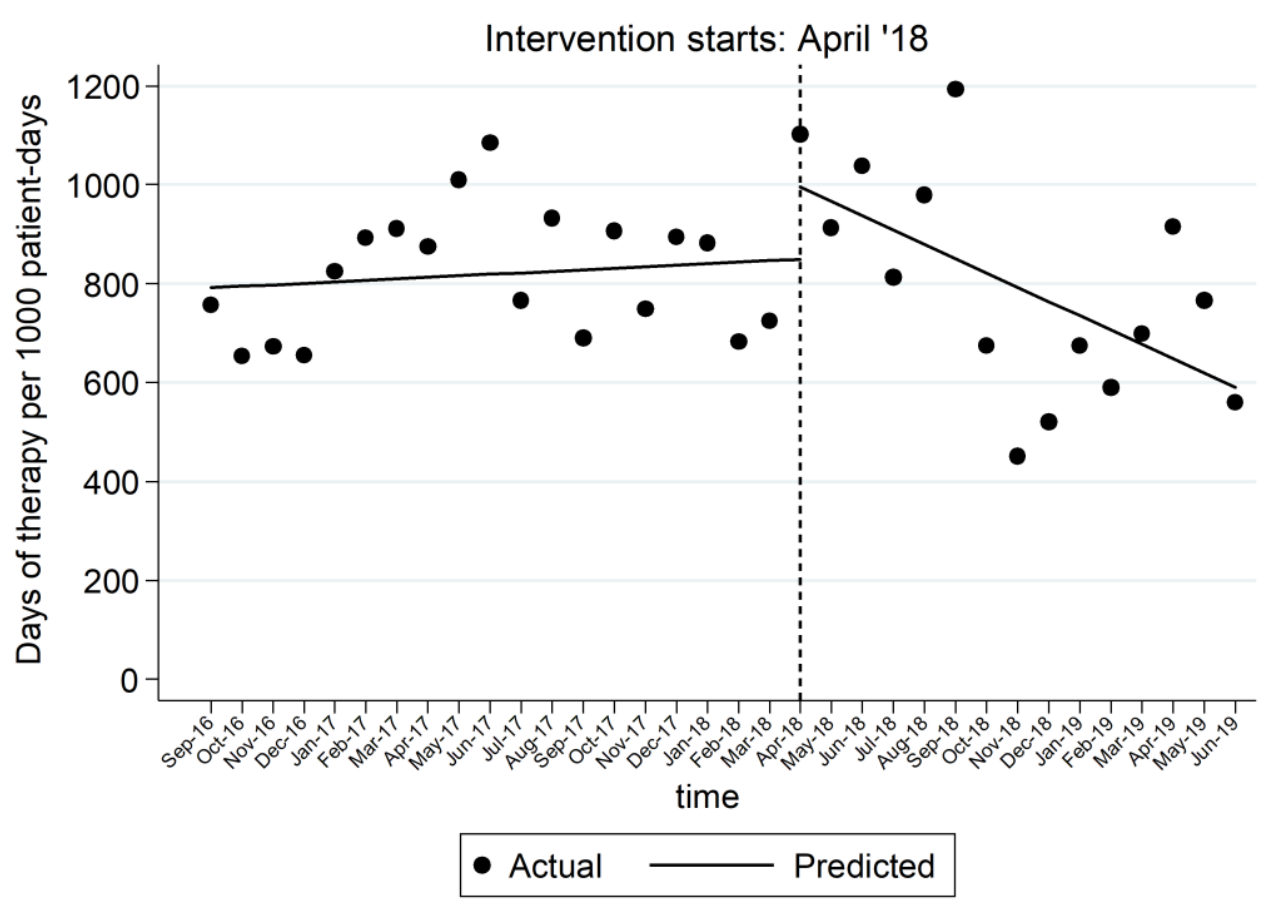

Figure 2. Interrupted time-series analysis of antibiotic use during the study in 13 NICUs in Greece. The period from September 2016 to March 2018 represents the pre-intervention period and April 2018 to June 2019 the post-intervention period.

\subsection{Prescribing Patterns, Length of Stay and Mortality}

The interventions did not seem to alter the prescribing patterns in the units with regard to antibiotics selected for the treatment of EOS. For the cases that empiric treatment was discontinued within 5 days, ampicillin and aminoglycosides constituted more than $90 \%$ of the DOTs administered on the first day and throughout the course, both before and after the interventions.

Regarding the median length of stay in the seven units that discontinued antibiotics early, in two units there was a statistically significant decrease by 2 days $(p<0.001$, $p=0.043$ ). In four units the median LOS decreased by 1 to 3 days, and in one unit the median LOS increased by 1 day.

Finally, in the seven units that increased discontinuation by day 5 , there were two deaths recorded among 292 cases in the pre-intervention period (6.8 deaths/1000cases) compared to one death among 309 cases (3.2 deaths/1000 cases) in the post-intervention period.

\section{Discussion}

Antibiotics are frequently prescribed in neonates for suspected EOS, even though the real risk is low. In view of antibiotics' adverse outcomes and increased risk for infection with multidrug-resistant pathogens, antimicrobial stewardship in the NICU is important. In a country with high overall antimicrobial use and resistance rates, stewardship initiatives are needed immediately, and at the same time they need to be adapted to work in a resource-limited healthcare system. The main study findings indicate that an antibiotic stewardship intervention using a "low-hanging fruit approach" can reduce the length of antibiotic therapy in low-risk neonates treated for possible EOS in Greek NICUs. A simple intervention, in which data on antibiotic use were shared with medical staff and a goal was established to stop antibiotic therapy by day 5 , led to an overall reduction of 615 antibiotic days / 1000 neonates within 15 months. Interrupted time series analysis revealed a declining trend in antibiotic consumption in the post-intervention period. Participating units that increased discontinued antibiotic regimens by day 5 showed a moderate but statistically 
significant increase in the actual number of neonates that received $\leq 5$ days of antibiotics in the post intervention period from $52.5 \%$ before to $61.6 \%$ after the intervention $(p=0.003)$.

Due to differences in methodology in the available literature, it is difficult to compare our results with similar studies involving stewardship interventions in NICUs [16]. Existing studies were mostly performed in single NICUs, recruited mixed populations (some including both EOS and late onset sepsis [11], and did not select comparable inclusion criteria or outcome measures.

A variety of known stewardship methodologies have been used in previous efforts to promote judicious antimicrobial use in NICUs. Revision or introduction of guidelines, prospective audit and feedback, pre-authorization, automatic stop orders and multidisciplinary rounds [11,17-26], have all been successful in lowering consumption according to the researchers' targets. Nonetheless, in some cases, stewardship interventions do not lead to shorter duration of therapy, even if additional diagnostics are used [27]. Successful stewardship initiatives are often supported by multidisciplinary teams consisting of pharmacists, infectious diseases specialists and microbiologists [28-30]. In this multicenter study, resource-demanding stewardship methods were not an option, and the support by dedicated multidisciplinary teams in each center was impossible. Consequently, the targets chosen were on a higher level, and followed a low-hanging fruit approach, with the main leverage for change being the periodic reporting of antimicrobial use per unit and benchmarking with other units.

Successful reduction in the use of specific targeted agents such as vancomycin, meropenem or cefotaxime has also been documented in the literature for antimicrobial stewardship efforts in NICUs [17-19,31,32]. This study did not have such an aim, and as such, similar results were not identified. There was a concern that the clinicians could adopt a more aggressive prescribing pattern in view of early discontinuation. However, agents used for empiric treatment did not change during the study period, as almost all units used exclusively ampicillin with an aminoglycoside as per guidelines [33].

Duration of empiric treatment for possible EOS in the NICUs showed significant variability This is actually a common finding that has been previously reported [7,12,15,34]. The median duration of antibiotics ranged from 2 to 8 days in our population. One previous study estimated a median of 7 days and a range of 5-14 days for cases of pneumonia, despite sterile cultures and cases of culture-negative sepsis [11]. Furthermore, in a cohort of clinically well infants who were feeding by $24 \mathrm{~h}$ of life, duration of treatment ranged from 1 to 10 days; $11.6 \%$ of them received antibiotics for 7-10 days even though they had negative cultures and regardless of risk factors [35].

Unnecessary exposure to extended courses of antibiotic regimens in NICUs is common practice, despite good evidence that symptoms encountered in neonatal sepsis have several mimickers [6]. Stewardship interventions leading to profound declines in overall antibiotic use (up to $27 \%$ ), even when including all admissions, have previously been described. In this case a 48 h electronic "hard stop" of antibiotics embedded in the electronic health record was used [11]. This is a clear indication that the magnitude of improvement in antibiotic use is linked to the organization and resources applied in ASPs. In the present study, the intervention was used in an environment of high antibiotic use, with restricted resources and a favorable outcome could be the stepping stone for further initiatives.

Why the "low-hanging fruit approach"?

For the purposes of this study, it was decided to intervene in a group of patients that were given antibiotics without appropriate indication according to national and international practice guidelines and failed to stop within $48 \mathrm{~h}$. Although most of this study's findings in terms of antibiotic overuse are relevant to countries with similar prescribing characteristics, the idea of identifying an achievable initial target before proceeding to other interventions, is applicable to all settings. The term "low-hanging fruit approach" refers to a selection of interventions that can be successful with limited resources and are easily attainable. This could involve switching antibiotics from intravenous to oral 
administration, stopping antibiotics early, or finding the common diagnosis linked with antibiotic overuse and developing a clinical pathway [14].

As antibiotic stewardship interventions require significant resources, complex organization and infrastructure, a full-scale program is often difficult to develop, especially in institutions where a dedicated team has not been established. In this context, choosing an easily achievable target such as establishing standardized, shorter antibiotic courses could lead to further interventions and successful outcomes. The unique environment of an intensive care unit is also important to consider. Neonatologists have a low threshold for obtaining cultures and starting antibiotics when they feel it is clinically relevant [9]. Taking into account this characteristic, this approach was chosen as a simple, feasible first goal that would also allow us to save resources and expand the program nation-wide.

Despite the limitation of automated data collection due to the lack of electronic health records, we managed to establish a national surveillance mechanism of antibiotic use in NICUs and to produce comparable data that allowed for benchmarking and identification of improvement targets.

This study has several limitations. First, we cannot be certain of the amount of blood drawn for the blood cultures taken, and we cannot account for variations in practice among units. Data on antibiotic consumption was collected in the NICUs using DOTs only for the first 7 days since the initiation of empiric treatment, while simultaneously measuring total length of therapy for each case. In the context of this study, the 7-day DOT approach could underestimate the effects of the intervention. Finally, it was not possible to measure readmission rates as a secondary outcome following our intervention, since after discharge, neonates may return to hospital on the general pediatric wards and not necessarily their local hospital.

Despite these limitations, a significant reduction of antibiotic therapy practices was documented within the network. Existing literature for this population suggests that further interventions can be applied [33,36]. However, these initial benefits could be reversed with time if sustainability of surveillance data collection and stewardship efforts cannot be ensured; a known trend previously described in pediatric antimicrobial stewardship initiatives [37]. Through this work, awareness has been raised for the need and importance of a collaborative network that collects benchmark data for quality improvement initiatives. This type of network has the potential to lead to a prolonged support of these efforts until more resources can be identified.

\section{Materials and Methods}

\subsection{Study Design and Population}

A nationwide prospective quasi-experimental study was performed, where 15 out of 17 public NICUs of the Greek National Health System contributed data between September 2016 and June 2019 after receiving ethics approval from their local authorities. Demographic, clinical, laboratory, and antibiotic consumption data were captured in an online database during the study period. Data were validated using automated algorithms and contact with the participating units when needed.

During the pre-intervention period (Sep 2016-Mar 2018), participating NICUs were asked to report the first 15 antibiotic regimens given each month, including those given within the $72 \mathrm{~h}$ of life for presumed early-onset sepsis (EOS), in order to explore possible targets for improvement. After reviewing these data, a group of neonates that were given prolonged antibiotic courses without sufficient risk factors for infection was identified. Neonates with the following characteristics formed the target group for the intervention: gestational age $\geq 37$ weeks, no evidence of clinical sepsis, CRP $\leq 10 \mathrm{mg} / \mathrm{L}$ during the first $72 \mathrm{~h}$ of life and negative cultures obtained within the first 3 days of antibiotic administration.

\subsection{Intervention}

In April 2018, participating NICUs received a complete analysis of the data collected and agreed to set the goal of antibiotic discontinuation within 5 days for neonates fulfilling 
the characteristics mentioned above. Two units decided not to move into the intervention phase. During the post-intervention period (April 2018-June 2019), data collection was adjusted to capture all cases treated for possible EOS. There were no other exclusion criteria, nor was it expected for participating units to change their practice or conduct specific lab exams at set time points. Additionally, it was up to the physicians' clinical judgement to decide if they would stop the antibiotics.

\subsection{Evaluation of Impact}

To evaluate the impact of the intervention for each unit, the length of therapy (LOT) (the number of days the neonate was receiving at least one antibiotic) was calculated, as well as length of stay (LOS) for the selected cases and the percentage of discontinued antibiotic regimens by day 5. Additionally, the mean length of therapy was calculated and used to estimate the gain or loss of antibiotic days by multiplying the difference of the means before and after the intervention with the number of neonates that met the criteria in the post-intervention period. The network's overall change in consumption was expressed per 1000 neonates for the post-intervention period. Days of therapy (DOT) per 1000 patient-days were used to assess trends of antibiotic consumption. DOT was defined as the aggregate sum of the days of exposure to each antibiotic on a 7-day follow-up from the initiation of empiric treatment. Each antibiotic for each day administered contributed by 1 . For example, a neonate that was on ampicillin and gentamicin for 5 days would have an LOT of 5 days, but a DOT of 10, as each of the two antibiotics was given for 5 days. Death before discharge was also followed up through medical records. Our manuscript follows SQUIRE 2.0 guidelines [38].

\subsection{Statistical Analysis}

Categorical data are presented in absolute and relative (\%) frequencies, while continuous data are presented with mean, standard deviation, median and interquartile range (IQR). Chi-square tests of independence were used to compare demographic characteristics before and after the intervention period, as well as the Mann-Whitney test, since normality of continuous data did not hold (tested with histograms). The Mann-Whitney test was also performed to compare the length of stay of neonates pre- and post-intervention (non-normal distribution). Interrupted time series analysis was used to establish whether there was a change in trend of antibiotic consumption after the implementation of the intervention. Results are presented as $\beta$-coefficients and $95 \%$ confidence intervals of the antibiotic use rate change. Statistical significance (a) was set to 5\%. All analyses were performed with STATA v.13.

\section{Conclusions}

Protecting neonates from prolonged and unnecessary antimicrobial exposure constitutes a public health priority. Fully developed stewardship interventions require multiple resources in terms of personnel and financial support making them difficult to implement. Adapting stewardship practices to local needs improves outcome and encourages practicing teams to participate. Following a "low-hanging fruit approach", a significant reduction in antibiotic use in a large network of NICUs was achieved. Implementing similar low-cost and low-resource actions could be successful in settings of high antibiotic consumption.

Author Contributions: Conceptualization, I.K., T.Z. and N.S.; Data curation, N.-M.M. and E.K.; Formal analysis, N.-M.M., E.K. and G.K. (Georgia Kourlaba); Funding acquisition, T.Z.; Investigation, PHiG, I.K., G.-C.T., E.B., E.C. (Elisavet Chorafa), V.G., D.G., T.G., A.K., K.K., G.K. (Georgia Karavana), M.L., V.P., M.P., E.R., T.S., E.S., G.A.S., C.T., M.N.T. and E.T.; Methodology, I.K., G.-C.T., N.-M.M., E.C. (Evangelia Chorianopoulou), E.K., G.K. (Georgia Kourlaba), C.T.,T.Z. and N.S.; Supervision, T.Z.; Validation, N.-M.M.; Visualization, I.K., N.-M.M. and E.K.; Writing—original draft, I.K. and N.S.; Writing-review \& editing, I.K., G.-C.T., N.-M.M., E.B., E.C. (Elisavet Chorafa), E.C. (Evangelia Chorianopoulou), V.G., D.G., T.G., A.K., K.K., G.K. (Georgia Karavana), E.K., G.K. (Georgia Kourlaba), 
M.L., V.P., M.P., E.R., T.S., E.S., G.A.S., C.T., M.N.T., E.T. and T.Z. All authors have read and agreed to the published version of the manuscript.

Funding: This research is part of a program that was funded by a grant of the Stavros Niarchos Foundation. The Stavros Niarchos Foundation had no involvement in the study design, the collection, analysis and interpretation of data, in the writing of the report or in the decision to submit the article for publication.

Institutional Review Board Statement: The study was conducted according to the guidelines of the Declaration of Helsinki, and approved by the Institutional Review Board (or Ethics Committee) of each hospital separately.

Informed Consent Statement: Informed consent was waived due to the nature and design of the study, and anonymized data collection.

Data Availability Statement: The datasets generated and/or analyzed during the current study are being used for further research and are available from the corresponding author on reasonable request.

Acknowledgments: The authors would like to thank Madeline E. Collins for editing and proofreading the manuscript.

Conflicts of Interest: The authors declare no conflict of interest. The funders had no role in the design of the study; in the collection, analyses, or interpretation of data; in the writing of the manuscript, or in the decision to publish the results.

\section{References}

1. Goossens, H.; Ferech, M.; Vanderstichele, R.; Elseviers, M. Outpatient Antibiotic Use in Europe and Association with Resistance: A Cross-National Database Study. Lancet 2005, 365, 579-587. [CrossRef]

2. Plachouras, D.; Kavatha, D.; Antoniadou, A.; Giannitsioti, E.; Poulakou, G.; Kanellakopoulou, K.; Giamarellou, H. Dispensing of Antibiotics without Prescription in Greece, 2008: Another Link in the Antibiotic Resistance Chain. Eurosurveillance 2010, $15,19488$. [CrossRef]

3. European Centre for Disease Prevention and Control. Antimicrobial Resistance in the EU/EEA (EARS-Net)—Annual Epidemiological Report for 2019; ECDC: Stockholm, Sweden, 2020.

4. Miyakis, S.; Pefanis, A.; Tsakris, A. The Challenges of Antimicrobial Drug Resistance in Greece. Clin. Infect. Dis. 2011, 53, 177-184. [CrossRef] [PubMed]

5. Cassini, A.; Högberg, L.D.; Plachouras, D.; Quattrocchi, A.; Hoxha, A.; Simonsen, G.S.; Colomb-Cotinat, M.; Kretzschmar, M.E.; Devleesschauwer, B.; Cecchini, M.; et al. Attributable Deaths and Disability-Adjusted Life-Years Caused by Infections with Antibiotic-Resistant Bacteria in the EU and the European Economic Area in 2015: A Population-Level Modelling Analysis. Lancet Infect. Dis. 2019, 19, 56-66. [CrossRef]

6. Gkentzi, D.; Dimitriou, G. Antimicrobial Stewardship in the Neonatal Intensive Care Unit: An Update. CPR 2019, 15, 47-52. [CrossRef] [PubMed]

7. Schulman, J.; Profit, J.; Lee, H.C.; Dueñas, G.; Bennett, M.V.; Parucha, J.; Jocson, M.A.L.; Gould, J.B. Variations in Neonatal Antibiotic Use. Pediatrics 2018, 142, e20180115. [CrossRef] [PubMed]

8. Cotten, C.M.; Taylor, S.; Stoll, B.; Goldberg, R.N.; Hansen, N.I.; Sanchez, P.J.; Ambalavanan, N.; Benjamin, D.K.; for the NICHD Neonatal Research Network. Prolonged Duration of Initial Empirical Antibiotic Treatment Is Associated with Increased Rates of Necrotizing Enterocolitis and Death for Extremely Low Birth Weight Infants. Pediatrics 2009, 123, 58-66. [CrossRef]

9. Cantey, J.B.; Patel, S.J. Antimicrobial Stewardship in the NICU. Infect. Dis. Clin. N. Am. 2014, 28, 247-261. [CrossRef] [PubMed]

10. Esaiassen, E.; Fjalstad, J.W.; Juvet, L.K.; van den Anker, J.N.; Klingenberg, C. Antibiotic Exposure in Neonates and Early Adverse Outcomes: A Systematic Review and Meta-Analysis. J. Antimicrob. Chemother. 2017, 72, 1858-1870. [CrossRef] [PubMed]

11. Cantey, J.B.; Wozniak, P.S.; Pruszynski, J.E.; Sánchez, P.J. Reducing Unnecessary Antibiotic Use in the Neonatal Intensive Care Unit (SCOUT): A Prospective Interrupted Time-Series Study. Lancet Infecti. Dis. 2016, 16, 1178-1184. [CrossRef]

12. Fjalstad, J.W.; Stensvold, H.J.; Bergseng, H.; Simonsen, G.S.; Salvesen, B.; Rønnestad, A.E.; Klingenberg, C. Early-Onset Sepsis and Antibiotic Exposure in Term Infants: A Nationwide Population-Based Study in Norway. Pediatr. Infect. Dis. J. 2015, 1. [CrossRef]

13. Russell, A.B.; Sharland, M.; Heath, P.T. Improving Antibiotic Prescribing in Neonatal Units: Time to Act. Arch. Dis. Child Fetal Neonatal. Ed. 2012, 97, F141-F146. [CrossRef]

14. Goff, D.A.; Bauer, K.A.; Reed, E.E.; Stevenson, K.B.; Taylor, J.J.; West, J.E. Is the "Low-Hanging Fruit" Worth Picking for Antimicrobial Stewardship Programs? Clin. Infect. Dis. 2012, 55, 587-592. [CrossRef]

15. Klingenberg, C.; Kornelisse, R.F.; Buonocore, G.; Maier, R.F.; Stocker, M. Culture-Negative Early-Onset Neonatal Sepsis-At the Crossroad between Efficient Sepsis Care and Antimicrobial Stewardship. Front. Pediatr. 2018, 6. [CrossRef] [PubMed]

16. Araujo da Silva, A.R.; Marques, A.; Di Biase, C.; Faitanin, M.; Murni, I.; Dramowski, A.; Hübner, J.; Zingg, W. Effectiveness of Antimicrobial Stewardship Programmes in Neonatology: A Systematic Review. Arch. Dis. Child 2020. [CrossRef] [PubMed] 
17. Chiu, C.-H.; Michelow, I.C.; Cronin, J.; Ringer, S.A.; Ferris, T.G.; Puopolo, K.M. Effectiveness of a Guideline to Reduce Vancomycin Use in the Neonatal Intensive Care Unit. Pediatr. Infect. Dis. J. 2011, 30, 273-278. [CrossRef] [PubMed]

18. Ting, J.Y.; Paquette, V.; Ng, K.; Lisonkova, S.; Hait, V.; Shivanada, S.; Tilley, P.; Osiovich, H.; Roberts, A. Reduction of Inappropriate Antimicrobial Prescriptions in a Tertiary Neonatal Intensive Care Unit After Antimicrobial Stewardship Care Bundle Implementation. Pediatr. Infect. Dis. J. 2019, 38, 54-59. [CrossRef]

19. Nzegwu, N.I.; Rychalsky, M.R.; Nallu, L.A.; Song, X.; Deng, Y.; Natusch, A.M.; Baltimore, R.S.; Paci, G.R.; Bizzarro, M.J. Implementation of an Antimicrobial Stewardship Program in a Neonatal Intensive Care Unit. Infect. Control Hosp. Epidemiol. 2017, 38, 1137-1143. [CrossRef] [PubMed]

20. Lee, I.; Fishman, N.O.; Zaoutis, T.E.; Morales, K.H.; Weiner, M.G.; Synnestvedt, M.; Nachamkin, I.; Lautenbach, E. Risk Factors for Fluconazole-Resistant Candida Glabrata Bloodstream Infections. Arch. Intern. Med. 2009, 169, 379. [CrossRef]

21. Bassiouny, D.M.; Hassan, R.M.; Shalaby, A.; Halim, M.M.A.; Wassef, M.A. Establishment of an Antimicrobial Stewardship Strategy on the Surgical NICU at Cairo University Specialized Pediatric Hospital. J. Pediatr. Surg. 2020, 55, 1959-1964. [CrossRef]

22. Tolia, V.; Desai, S.; Qin, H.; Rayburn, P.; Poon, G.; Murthy, K.; Ellsbury, D.; Chiruvolu, A. Implementation of an Automatic Stop Order and Initial Antibiotic Exposure in Very Low Birth Weight Infants. Am. J. Perinatol. 2016, 34, 105-110. [CrossRef]

23. Lu, C.; Liu, Q.; Yuan, H.; Wang, L. Implementation of the Smart Use of Antibiotics Program to Reduce Unnecessary Antibiotic Use in a Neonatal ICU: A Prospective Interrupted Time-Series Study in a Developing Country. Crit. Care Med. 2019, 47, e1-e7. [CrossRef]

24. El-Baky, R.M.A.; Senosy, E.M.; Omara, W.; Mohamed, D.S.; Ibrahim, R.A. The Impact of the Implementation of Culture-Based Antibiotic Policy on the Incidence of Nosocomial Infections in Neonates Hospitalized in Neonatal Intensive Care Unit in a General Egyptian Hospital in Upper Egypt, 2016-2018. J. Pure Appl. Microbiol. 2020, 14, 1879-1892. [CrossRef]

25. Thampi, N.; Shah, P.S.; Nelson, S.; Agarwal, A.; Steinberg, M.; Diambomba, Y.; Morris, A.M. Prospective Audit and Feedback on Antibiotic Use in Neonatal Intensive Care: A Retrospective Cohort Study. BMC Pediatr. 2019, 19, 105. [CrossRef]

26. Gustavsson, L.; Lindquist, S.; Elfvin, A.; Hentz, E.; Studahl, M. Reduced Antibiotic Use in Extremely Preterm Infants with an Antimicrobial Stewardship Intervention. BMJ Paediatr. Open 2020, 4, e000872. [CrossRef]

27. Dretvik, T.; Solevåg, A.L.; Finvåg, A.; Størdal, E.H.; Størdal, K.; Klingenberg, C. Active Antibiotic Discontinuation in Suspected but Not Confirmed Early-Onset Neonatal Sepsis-A Quality Improvement Initiative. Acta Paediatr. 2020, 109, 1125-1130. [CrossRef] [PubMed]

28. Dyar, O.J.; Tebano, G.; Pulcini, C. Managing Responsible Antimicrobial Use: Perspectives across the Healthcare System. Clin. Microbiol. Infect. 2017, 23, 441-447. [CrossRef]

29. Carling, P.; Fung, T.; Killion, A.; Terrin, N.; Barza, M. Favorable Impact of a Multidisciplinary Antibiotic Management Program Conducted During 7 Years. Infect. Control Hosp. Epidemiol. 2003, 24, 699-706. [CrossRef] [PubMed]

30. Bouchet, F.; Le Moing, V.; Dirand, D.; Cros, F.; Lienard, A.; Reynes, J.; Giraudon, L.; Morquin, D. Effectiveness and Acceptance of Multimodal Antibiotic Stewardship Program: Considering Progressive Implementation and Complementary Strategies. Antibiotics 2020, 9, 848. [CrossRef] [PubMed]

31. Hamdy, R.F.; Bhattarai, S.; Basu, S.K.; Hahn, A.; Stone, B.; Sadler, E.D.; Hammer, B.M.; Galiote, J.; Slomkowski, J.; Casto, A.M.; et al. Reducing Vancomycin Use in a Level IV NICU. Pediatrics 2020, 146. [CrossRef] [PubMed]

32. Astorga, M.C.; Piscitello, K.J.; Menda, N.; Ebert, A.M.; Ebert, S.C.; Porte, M.A.; Kling, P.J. Antibiotic Stewardship in the Neonatal Intensive Care Unit: Effects of an Automatic 48-Hour Antibiotic Stop Order on Antibiotic Use. J. Pediatr. Infect. Dis. Soc. 2019, 8, 310-316. [CrossRef]

33. Puopolo, K.M.; Benitz, W.E.; Zaoutis, T.E.; Committee on Fetus and Newborn; Committee on Infectious Diseases. Management of Neonates Born at $\geq 35$ 0/7 Weeks' Gestation with Suspected or Proven Early-Onset Bacterial Sepsis. Pediatrics 2018, 142, e20182894. [CrossRef]

34. Schulman, J.; Dimand, R.J.; Lee, H.C.; Duenas, G.V.; Bennett, M.V.; Gould, J.B. Neonatal Intensive Care Unit Antibiotic Use. Pediatrics 2015, 135, 826-833. [CrossRef]

35. Spitzer, A.R.; Kirkby, S.; Kornhauser, M. Practice Variation in Suspected Neonatal Sepsis: A Costly Problem in Neonatal Intensive Care. J. Perinatol. 2005, 25, 265-269. [CrossRef] [PubMed]

36. Ho, T.; Dukhovny, D.; Zupancic, J.A.F.; Goldmann, D.A.; Horbar, J.D.; Pursley, D.M. Choosing Wisely in Newborn Medicine: Five Opportunities to Increase Value. Pediatrics 2015, 136, e482-e489. [CrossRef]

37. Gerber, J.S.; Prasad, P.A.; Fiks, A.G.; Localio, A.R.; Bell, L.M.; Keren, R.; Zaoutis, T.E. Durability of Benefits of an Outpatient Antimicrobial Stewardship Intervention After Discontinuation of Audit and Feedback. JAMA 2014, 312, 2569. [CrossRef] [PubMed]

38. Ogrinc, G.; Davies, L.; Goodman, D.; Batalden, P.; Davidoff, F.; Stevens, D. SQUIRE 2.0 (Standards for QUality Improvement Reporting Excellence): Revised Publication Guidelines from a Detailed Consensus Process. BMJ Qual. Saf. 2016, 25, 986-992. [CrossRef] [PubMed] 